\title{
Nonlinear matching of Solitons - Continued redshift between silica and soft-glass fibers
}

\author{
Agger, Christian; Sørensen, Simon Toft; Thomsen, Carsten ; Keiding, Søren ; Bang, Ole
}

Published in:

CLEO Technical Digest

Publication date:

2012

Document Version

Publisher's PDF, also known as Version of record

Link back to DTU Orbit

Citation (APA):

Agger, C., Sørensen, S. T., Thomsen, C., Keiding, S., \& Bang, O. (2012). Nonlinear matching of Solitons Continued redshift between silica and soft-glass fibers. In CLEO Technical Digest (pp. QF1G.8). Optical Society of America.

\section{General rights}

Copyright and moral rights for the publications made accessible in the public portal are retained by the authors and/or other copyright owners and it is a condition of accessing publications that users recognise and abide by the legal requirements associated with these rights.

- Users may download and print one copy of any publication from the public portal for the purpose of private study or research.

- You may not further distribute the material or use it for any profit-making activity or commercial gain

- You may freely distribute the URL identifying the publication in the public portal 


\title{
Nonlinear matching of Solitons - Continued redshift between silica and soft-glass fibers.
}

\author{
Christan Agger $^{1}$, Simon Sørensen ${ }^{1}$, Carsten Thomsen ${ }^{3}$, Søren Keiding ${ }^{2}$, and Ole Bang ${ }^{1}$. \\ DTU Fotonik, Department of Photonics Engineering, Tech. Uni. of Denmark, 2800 Kgs. Lyngby, Denmark ${ }^{1}$. \\ Department of Chemistry, Aarhus University, Langelandsgade 140, 8000 Aarhus C, Denmark ${ }^{2}$. \\ NKT Photonics, Blokken 84, 3460 Birkerød, Denmark ${ }^{3}$. \\ cagg@fotonik.dtu.dk
}

\begin{abstract}
We present an analysis of nonlinear coupling between fibers. We introduce the nonlinear coupling coefficient and investigate solitons coupling from one fiber into another. We will also present simulated supercontinuum from concatenated fiber systems.
\end{abstract}

(C) 2011 Optical Society of America

OCIS codes: $190.4370,190.5650$.

In the search for making a versatile broadband infrared (IR) supercontinuum (SC) source, the dynamics of soliton coupling between optical fibers is of great interest. Soft-glass fibers made from, e.g., ZBLAN or tellurite, are promising candidates as nonlinear fibers used in IR SC sources for broadening the spectrum of light beyond the material loss limit of silica around $2.5 \mu \mathrm{m}$. Generally, soft-glass fibers have zero dispersion wavelengths (ZDW) at longer wavelengths than silica fibers, and thus systems of concatenated silica and soft-glass fibers have been proposed to enable the use of $\mathrm{Er} / \mathrm{Yr}$ based lasers for anomalous dispersion regime pumping [1-4]. In such concatenated systems, solitons redshift, by the soliton self-frequency shift (SSFS), initially in a silica fiber (fiber 1) until strong dispersion prevents further redshift [4]. Then, light is coupled into a soft-glass fiber (fiber 2) for further redshifting into the IR.

When coupling linear waves between optical waveguides, mode-field diameter matching is important to avoid loss of power. When coupling fundamental solitons between two nonlinear fibers however, soliton number preservation becomes an equally important matching requirement, placing restrictions not only on the group velocity dispersion of the fibers $\left(\beta_{2}\right)$, but also on their nonlinearity $\gamma=n_{2} \omega /\left(c A_{\mathrm{ff}}\right)[5,6]$. Here $n_{2}$ is the nonlinear refractive index of the fiber material, $\omega$ is the frequency, $c$ is the speed of light in vacuum and $A_{\mathrm{eff}}$ is the effective area of the mode. There are two scenarios, in which nonlinear processes dominate further dynamics after coupling of light into fiber 2 , and both are determined by the soliton number in fiber $2, N_{2}$. (1) If $1 / 2<N_{2}<3 / 2$ (Fig. 1 left panel, top left) the coupled light will continue propagation as a fundamental soliton in fiber 2. (2) If $N_{2}>3 / 2$ (Fig. 1, left panel, top right) a higher order soliton is generated, which will undergo soliton fission and possibly generate dispersive waves.

As a numerical example illustrating the nonlinear soliton matching (NLSM), we consider coupling between silica and soft-glass photonic crystal fibers (PCFs) with hole diameter $d$, pitch $\Lambda$, and 7 rings of air-holes. All fiber properties
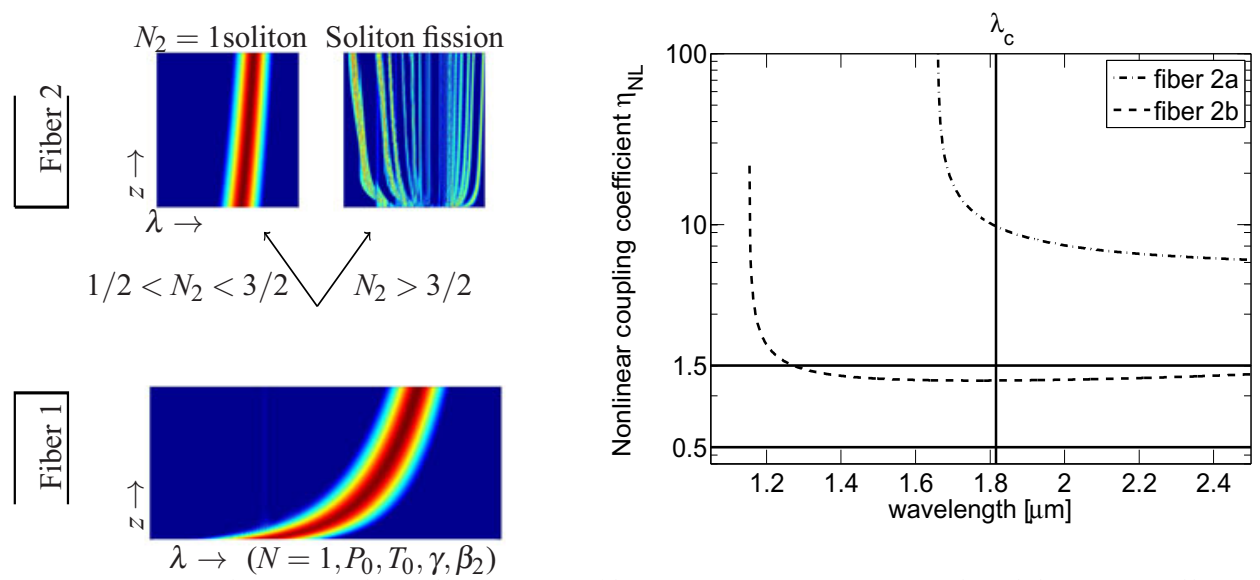

Fig. 1. Left panel: Spectral evolution in a fiber system for continued redshift: Initially an $N=1$ soliton redshifts in fiber 1 (silica), and the output is coupled into fiber 2 (soft-glass). Right panel: Nonlinear coupling coefficient, $\eta_{\mathrm{NL}}$ for fiber $2 \mathrm{a}$ (dash dotted) and fiber $2 \mathrm{~b}$ (dashed) with $\eta=1$. 

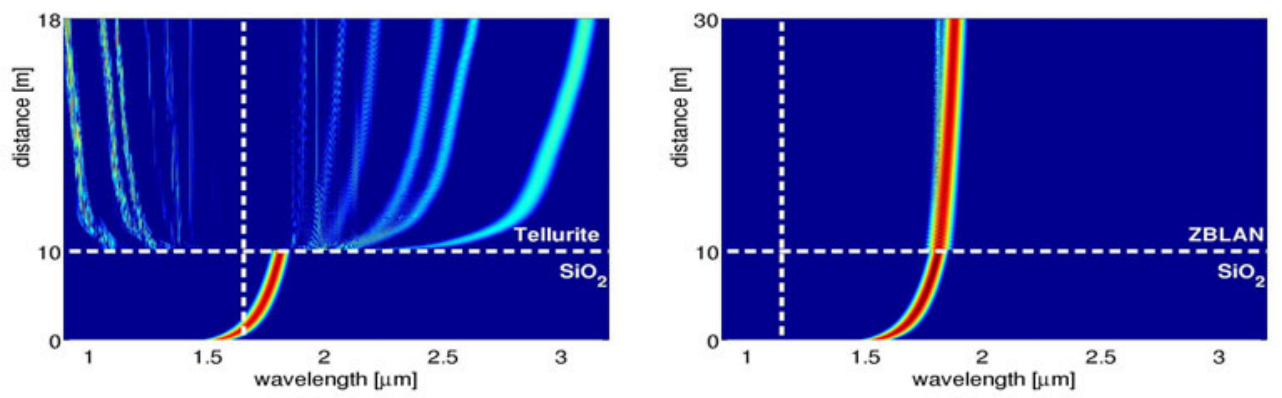

Fig. 2. Spectral Evolution in fiber 1 and fiber $2 \mathrm{a}$ (left panel) and fiber 1 and fiber $2 \mathrm{~b}$ (right panel). Left panel: $\eta_{\mathrm{NL}} \gg 3 / 2$ and a higher order soliton undergoes soliton fission. Right panel: $1 / 2<\eta_{\mathrm{NL}}<3 / 2$, and a single fundamental soliton continues propagation. White vertical line marks $\lambda_{\mathrm{ZD}}$ of fiber 2 .

are calculated using a commercially available finite-element tool and material dispersion taken from the literature. Fiber 1 is a PCF $(d=3.3 \mu \mathrm{m}$ and $\Lambda=6.35 \mu \mathrm{m})$ made from fused silica and we investigate two different materials for fiber 2. Fiber $2 \mathrm{a}$ is a PCF $(d=3.5 \mu \mathrm{m}, \Lambda=4.38 \mu \mathrm{m})$ made from highly nonlinear tellurite and fiber $2 \mathrm{~b}$ is a fluoride based ZBLAN PCF ( $d=3.3 \mu \mathrm{m}$ and $\Lambda=6.35 \mu \mathrm{m})$. In optical fibers the equation governing the dynamics is the generalized nonlinear Schrödinger equation (GNLSE) which we numerically. As initial condition we use a fundamental soliton with a central wavelength $\lambda_{p}=1550 \mathrm{~nm}$, and FWHM pulse duration of $T_{\mathrm{FWHM}}=50 \mathrm{fs}$. After propagation in $10 \mathrm{~m}$ of fiber 1, simulation of the GNLSE shows that the soliton has redshifted to approximately $\lambda_{c}=1820 \mathrm{~nm}$. We define the nonlinear coupling coefficient as [5],

$$
\eta_{\mathrm{NL}}^{2} \equiv N_{2}^{2} / N_{1}^{2}=\eta\left(\gamma_{2} /\left|\beta_{22}\right|\right) /\left(\gamma_{1} /\left|\beta_{21}\right|\right)
$$

which is given in terms of material parameters through the nonlinear coefficient $\gamma_{i}$, and fiber properties through $\beta_{2 i}$ for the $i$-th fiber, respectively $(i=1,2)$. In Eq. (1) the power coupling efficiency $0 \leq \eta \leq 1$, accounts for Fresnel reflection, mode field diameter mismatch and similar loss mechanisms. In the numerical treatment we take $\eta=1$ [5]. In Fig. 1 right panel, we show $\eta_{\mathrm{NL}}$ for fiber $2 \mathrm{a}$ and $2 \mathrm{~b}$ as function of wavelength. Notice that $\eta_{\mathrm{NL}}$ is singular at $\beta_{22}=0$, thus $\eta_{\mathrm{NL}} \rightarrow \infty$ at $\lambda_{\mathrm{zD}}$ of fiber 2 . The black horizontal solid lines mark the band of NLSM, where continued SSFS of a stable fundamental soliton can be expected. We find that $\eta_{\mathrm{NL}} \gg 3 / 2$ for fiber $2 \mathrm{a}$, and consequently we expect soliton fission will occur in fiber $2 \mathrm{a}$ after coupling from fiber 1. Simulation results for propagation in $10 \mathrm{~m}$ of fiber 1 and $8 \mathrm{~m}$ in fiber 2a are shown in Fig. 2 left panel. Here it is clearly confirmed that after a short distance of propagation in fiber $2 \mathrm{a}$, soliton fission causes a breakup of the pulse, and generates multiple separated solitons. The central wavelength of the most redshifted soliton becomes approximately $\lambda=3100 \mathrm{~nm}$ in this case, and $\sim 18 \%$ of the energy in the input soliton has been converted to this wavelength. If, on the other hand, the output from fiber 1 is coupled into fiber $2 b$, where the NLSM condition is satisfied so that $1 / 2<\eta_{\mathrm{NL}}<3 / 2$, a fundamental soliton will continue propagation and redshift. This is also confirmed by simulations shown in Fig. 2 right panel. Here it is shown that the fundamental soliton from fiber 1 continues stable redshift in fiber $2 \mathrm{~b}$, and the central wavelength increases to $1857 \mathrm{~nm}$. In this case approximately $83 \%$ of the energy from the input pulse is converted to this wavelength.

In the presentation we will further present how the NLSM scheme can be used for optimum coupling of a broadband $\mathrm{SC}$ between silica and soft-glass fibers. Here we will focus on the importance of the bandwidth over which $\eta_{\mathrm{NL}}>1 / 2$ for optimum coupling.

\section{References}

1. C. L. Hagen, J. W. Walewski, and S. T. Sanders, Photonics Technology Letters, IEEE 18, 91 (2006).

2. C. Xia, M. Kumar, O. P. Kulkarni, M. N. Islam, J. Fred L. Terry, M. J. Freeman, M. Poulain, and G. Mazé, Opt. Lett. 31, 2553 (2006).

3. C. Xia, Z. Xu, M. N. Islam, F. L. Terry, M. J. Freeman, A. Zakel, and J. Mauricio, Selected Topics in Quantum Electronics, IEEE Journal of 15, 422 (2009).

4. J. C. Travers, S. V. Popov, and J. R. Taylor, Opt. Lett. 30, 3132 (2005).

5. C. Agger, S. T. Sørensen, C. L. Thomsen, S. R. Keiding, and O. Bang, Opt. Lett. 36, 2596 (2011).

6. D. Anderson, M. Lisak, B. Malomed, and M. Quiroga-Teixeiro, J. Opt. Soc. Am. B 11, 2380 (1994). 